\title{
BSREM for Brain Metastasis Detection with 18F-FDG-PET/CT in Lung Cancer Patients
}

\author{
Virginia Liberini ${ }^{1,2,3}$ - Daniele A. Pizzuto ${ }^{1,4} \cdot$ Michael Messerli $^{1}$. Erika Orita ${ }^{1,5} \cdot$ Hannes Grünig $^{1}$. \\ Alexander Maurer ${ }^{1}$. Cäcilia Mader ${ }^{1}$. Lars Husmann ${ }^{1}$. Désirée Deandreis ${ }^{2} \cdot$ Fotis Kotasidis $^{6}$. Josey Trinckauf ${ }^{1}$. \\ Alessandra Curioni ${ }^{7} \cdot$ Isabelle Opitz $^{8} \cdot$ Sebastian Winklhofer $^{9} \cdot$ Martin W. Huellner $^{1}$
}

Received: 10 July 2021 / Revised: 10 July 2021 / Accepted: 13 December 2021 / Published online: 25 February 2022

(c) The Author(s) 2022

\begin{abstract}
The aim of the study was to analyze the use of block sequential regularized expectation maximization (BSREM) with different $\beta$-values for the detection of brain metastases in digital fluorine-18 labeled 2-deoxy-2-fluoro-D-glucose (18F-FDG) PET/ $\mathrm{CT}$ in lung cancer patients. We retrospectively analyzed staging/restaging 18F-FDG PET/CT scans of 40 consecutive lung cancer patients with new brain metastases, confirmed by MRI. PET images were reconstructed using BSREM ( $\beta$-values of $100,200,300,400,500,600,700)$ and OSEM. Two independent blinded readers (R1 and R2) evaluated each reconstruction using a 4-point scale for general image quality, noise, and lesion detectability. SUVmax of metastases, brain background, target-to-background ratio (TBR), and contrast recovery (CR) ratio were recorded for each reconstruction. Among all reconstruction techniques, differences in qualitative parameters were analyzed using non-parametric Friedman test, while differences in quantitative parameters were compared using analysis of variances for repeated measures. Cohen's kappa $(k)$ was used to measure inter-reader agreement. The overall detectability of brain metastases was highest for BSREM200 (R1: $2.83 \pm 1.17 ; \mathrm{R} 2: 2.68 \pm 1.32$ ) and BSREM300 (R1: $2.78 \pm 1.23$; R2: $2.68 \pm 1.36$ ), followed by BSREM100, which had lower accuracy owing to noise. The highest median TBR was found for BSREM100 (R1: 2.19 $\pm 1.05 ; \mathrm{R} 2: 2.42 \pm 1.08$ ), followed by BSREM200 and BSREM300. Image quality ratings were significantly different among reconstructions $(p<0.001)$. The median quality score was higher for BSREM100-300, and both noise and metastases' SUVmax decreased with increasing $\beta$-value. Inter-reader agreement was particularly high for the detectability of photopenic metastases and blurring (all $k>0.65$ ). BSREM200 and BSREM300 yielded the best results for the detection of brain metastases, surpassing both BSREM400 and OSEM, typically used in clinical practice.
\end{abstract}

Keywords Lung cancer $\cdot$ Brain metastases $\cdot 18 \mathrm{~F}-\mathrm{FDG} \cdot \mathrm{PET} / \mathrm{CT} \cdot \mathrm{OSEM} \cdot \mathrm{BSREM}$

\section{Abbreviations}

18F-FDG Fluorine-18 labeled 2-deoxy-2-fluoro-D-glucose

AJCC American Joint Committee on Cancer

BMI Body mass index

Virginia Liberini

virginia.liberini@usz.ch

1 Department of Nuclear Medicine, University Hospital Zürich, University of Zürich, Zürich, Switzerland

2 Department of Medical Science, Unit of Nuclear Medicine, University of Turin, Turin, Italy

3 Nuclear Medicine Department, S. Croce E Carle Hospital, Cuneo, Italy

4 Nuclear Medicine Unit, Fondazione Policlinico Universitario A. Gemelli IRCCS, 00168 Rome, Italy
BPL Bayesian penalized likelihood

BSREM Block sequential regularized expectation maximization

CE Contrast-enhanced

CECT Contrast-enhanced computed tomography
5 Department of Radiology, Nippon Medical School, 1-1-5 Sendagi, Bunkyo-ku, Tokyo 113-8603, Japan

6 GE Healthcare, Waukesha, WI, USA

7 Department of Medical Oncology and Hematology, University Hospital Zurich, University of Zurich, Zurich, Switzerland

8 Department of Thoracic Surgery, University Hospital Zurich, Zurich, Switzerland

9 Department of Neuroradiology, Clinical Neuroscience Center, University Hospital Zurich, Zurich, Switzerland 


$\begin{array}{ll}\text { CR } & \text { Contrast recovery } \\ \text { CT } & \text { Computed tomography } \\ \text { MIP } & \text { Maximum intensity projection } \\ \text { MRI } & \text { Magnetic resonance imaging } \\ \text { MVXD } & \text { MultiVane-XD sequence } \\ \text { OSEM } & \text { Ordered subset expectation maximization } \\ \text { PET } & \text { Positron emission tomography } \\ \text { PSF } & \text { Point spread function } \\ \text { SiPM } & \text { Silicon photomultiplier } \\ \text { SUVmax } & \text { Maximum standardized uptake value } \\ \text { TBR } & \text { Target-to-background ratio } \\ \text { TFE } & \text { Turbo field echo } \\ \text { TOF } & \text { Time of flight } \\ \text { TSE } & \text { Turbo spin-echo } \\ \text { VOI } & \text { Volume of interest }\end{array}$

\section{Introduction}

Brain metastases (BM) are the most frequent intracranial tumors in adults. Lung cancer, breast cancer, melanoma, renal cell carcinoma (RCC), and colorectal cancer (CRC) are the most common solid tumors associated with BM. BM are associated with higher morbidity and mortality, independently of the type of primary tumor, with an overall survival of less than 2 years [1].

BM occur in approximately 10-20\% of lung cancer patients with metastatic disease and approximately 30-50\% of patients with non-small-cell lung cancer (NSCLC) will eventually develop BM [2-4]. BM are diagnosed more frequently in small-cell lung cancer (SCLC) compared to other types of lung cancer and are present at initial staging already in approximately $10 \%$ of patients [5, 6]. In NSCLC patients, adenocarcinoma subtype, advanced nodal status, advanced tumor stage, and young patient age are known risk factors for the metachronous development of BM [7-9]. The reported overall survival at 60 months is $68 \%$ in stage IB, while it is less than $10 \%$ in stage IVB [4].

Gadolinium-enhanced magnetic resonance imaging (MRI) is the gold standard for diagnosing BM non-invasively. Brain MRI is not recommended by guidelines for screening in the majority of solid tumors in the absence of suspicious clinical symptoms. In lung cancer, brain MRI is generally recommended for NSCLC stage III and IV [10, 11]. For lower NSCLC stages, recommendations in guidelines vary: while the ESMO guidelines acknowledge usefulness of MRI in stages I and II [12], MRI is considered optional in stage IB by the NCCN guidelines [13] and is encouraged in all stages if there is curative intent by the NICE guidelines [14]. In SCLC, brain MRI is generally recommended in all stages [13, 15].

The staging of lung cancer is among the most widely acknowledged indications for 2-deoxy-2-[18F]fluoro-D-glucose (18F-FDG) positron-emission tomography (PET)/computed tomography (CT) worldwide. It is considered particularly helpful for the detection of lymph node metastases and distant metastases [16]. In particular, 18F-FDG-PET/CT is recommended for staging NSCLC due to its excellent ability (sensitivity $93 \%$, specificity $96 \%$ ) to detect adrenal and bone metastases, if baseline CT is negative (level of evidence A). 18F-FDG-PET/ $\mathrm{CT}$ is also proposed in oligometastatic NSCLC patients potentially eligible for treatment. In SCLC patients, although data are still not sufficient (level of evidence C), 18F-FDG-PET/CT is also proposed for staging, particularly for the detection of bone metastases [17-19]. However, the detection rate of BM by $18 \mathrm{~F}-$ FDG PET/CT is consistently low, ranging between 1 and $2.1 \%$ [20-22]. This is owing to the low spatial resolution of PET on the one hand, and the high physiologic FDG avidity of brain background on the other hand, which leads to a low target-tobackground ratio (TBR) with poor contrast and poor delineation of cerebral lesions [23-26]. Nevertheless, 18F-FDG PET/CT scans may lead to an incidental detection of BM in patients in the absence of brain MRI, both in early NSCLC stages and in patients at restaging.

Novel iterative Bayesian penalized likelihood reconstruction algorithms, such as block sequential regularized expectation maximization (BSREM), have improved the detectability of small-sized, faintly FDG-avid lesions with low TBR, which holds true for a proportion of brain metastases [27-29]. With BSREM, the optimal reconstruction results depend on the global strength of the regularization term ( $\beta$-value) [30-33]. For whole-body 18F-FDG scans in oncology, the $\beta$-value is typically set to 400-450 [34-37]. This $\beta$-value range represents the clinical standard also at our institution. However, some preliminary data highlight that brain 18F-FDG image quality might benefit from different $\beta$-values in BSREM reconstruction [38].

The aim of our study was to compare block sequential regularized expectation maximization (BSREM) with different $\beta$-values and ordered subset expectation maximization (OSEM) algorithms, in order to define which reconstruction algorithm is most appropriate for brain metastases detection in digital 18F-FDG PET/CT.

\section{Material and Methods}

\section{Patient Selection}

We retrospectively analyzed a cohort of 492 consecutive patients, who underwent a clinically indicated $18 \mathrm{~F}-\mathrm{PET} / \mathrm{CT}$ scan on a digital scanner for the staging/restaging of lung cancer at the University Hospital of Zürich between May 2017 and January 2020.

The primary inclusion criteria for this retrospective observational study were (a) patient consent for the use of medical data for retrospective studies, (b) histologically proven lung cancer, (c) 18F-FDG PET/CT scan acquired on 
a digital scanner with silicon photomultiplier (SiPM) technology, and (d) new onset of brain metastases confirmed by gadolinium-enhanced MRI, performed within 30 days of PET/CT. Patients were excluded if (a) age $<18$ years old, (b) simultaneous presence of other clinically manifest tumor entity, and (c) no PET raw data available. Finally, out of the 492 patients, a total of 40 patients with MRI-confirmed brain metastases were included (refer to flow chart in supplemental fig. S1).

Our study was approved by the local ethics committee and was conducted in compliance with ICH-GCP rules and the Declaration of Helsinki.

\section{PET/CT Acquisition}

Patients fasted for at least $4 \mathrm{~h}$ prior to the scan, and blood glucose levels were below $160 \mathrm{mg} / \mathrm{dl}$ at the time of $18 \mathrm{~F}$ FDG injection. All patients underwent a PET/CT scan on a digital scanner with silicon photomultiplier (SiPM) technology (GE Discovery Molecular Insights-DMI PET/CT, GE Healthcare, Waukesha, WI). The injected tracer activity was $202.00 \pm 73.84 \mathrm{MBq}$ of $18 \mathrm{~F}-\mathrm{FDG}$. After an uptake time of $60 \mathrm{~min}$ and following $\mathrm{CT}$ acquisition both for attenuation correction and anatomical correlation (from the vertex of the skull to the mid-thighs or to the feet), PET data were acquired in 3-dimensional time-of-flight (TOF) mode, covering the identical anatomical region of the CT, with $2.5 \mathrm{~min} / \mathrm{bed}$ position and $6-11$ bed positions per patient ( $23 \%$ overlap), depending on patient size.

\section{Bayesian Penalized Likelihood Reconstruction Algorithms}

Ordered subset expectation maximization (OSEM) PET image reconstruction was introduced in 1994 and is still a widely used reconstruction algorithm for PET images. OSEM divides the image data into subsets, to whom an expectation maximization is applied, leading to less artifacts and image noise compared to older reconstruction algorithms [30, 31]. More recently, point spread function modeling was implemented with OSEM $\left(\mathrm{OSEM}_{\mathrm{PSF}}\right)$, which further improved the signal-to-noise ratio (SNR) [39, 40]. OSEM images with 25 iterations provide almost perfect SUV quantitation, but at the expense of severe image noise. Hence, OSEM is typically stopped after 2-4 iterations with subsequent inaccuracies in quantitative assessment [41-43].

Recently, the switch from large conventional photomultiplier tubes to small solid state silicon-based photomultipliers (SiPMs) lead to the improvement of several technical aspects: e.g., higher spatial resolution (3-4 mm pixels for digital versus 4-6 mm pixels for conventional system), higher geometric sensitivity (by removing collimation and using a longer axial FOV), and a high effective sensitivity owing to time-of-flight (TOF) measurements (from 500-600 to 200-300 ps) [44-47].

PET image reconstruction has so evolved further with the advent of Bayesian penalized likelihood (BPL) reconstruction algorithms, such as block sequential regularized expectation maximization (BSREM - Q.Clear; GE Healthcare). BPL reconstruction algorithms increase the accuracy of lesion quantitation compared to OSEM by maximizing signal-to-noise ratio (SNR), while achieving almost full convergence [48-50], yielding advantages in oncological populations [32, 51-53]. With BSREM, the optimal reconstruction results depend on the global strength of the regularization term ( $\beta$-value), which again depends on several aspects (e.g., dosage, noise, radionuclide used, anatomical area of examination) [30-33, 54].

\section{Image Reconstruction}

In all 40 patients with MRI-confirmed brain metastases, dedicated brain PET image datasets were reconstructed with different standardized settings (all with a $256 \times 256$ pixel matrix):

1- OSEM: 3 iterations, 16 subsets, FWHM of $6.3 \mathrm{~mm}$, 1:4 Z-axis filter and $6.4 \mathrm{~mm}$ Gaussian filter with both time-of-flight (TOF) and point spread function (PSF) modeling $\left(\mathrm{OSEM}_{\mathrm{PSF}}\right.$; VUE Point FX with SharpIR, GE Healthcare).

2- BSREM (Q.Clear, GE Healthcare) with both TOF and PSF and $\beta$-values of $100\left(\right.$ BSREM $\left._{100}\right), 200\left(\right.$ BSREM $\left._{200}\right), 300$ $\left(\right.$ BSREM $\left._{300}\right), 400\left(\right.$ BSREM $\left._{400}\right), 500\left(\right.$ BSREM $\left._{500}\right), 600$ $\left(\right.$ BSREM $\left._{600}\right), 700\left(\right.$ BSREM $\left._{700}\right)$.

\section{Qualitative Imaging Analysis}

A total of 320 reconstructed PET/CT datasets (40 patient studies, each with the eight aforementioned different reconstructions) were evaluated by two readers (D.P. and V.L, with 7 and 6 years of experience in nuclear medicine, respectively) blinded to the reconstruction method used. All scans were reviewed independently on a dedicated workstation (Advantage Workstation, Version 4.6; GE Healthcare) and in random order. Readers identified cerebral metastases by reading both PET and CT images. Readers were blinded to all clinical information, except the presence of brain metastases from a primary lung tumor. In case of discrepancy of image rating, a final decision was made by consensus including a third reader (MWH, with 11 years of experience in nuclear medicine and radiology).

Readers first rated the general image quality; for this purpose, datasets were viewed using axial views with reformatted sections. The two readers evaluated several qualitative aspects using a 4-point grading scale: (a) general image quality (GIq) 
score of each reconstructed image, based on the ability to discriminate grey matter (GM) from white matter (WM); (b) noise score; and (c) overall lesion detectability. Both readers assessed overall brain GIq and noise score as well as neocortex, basal ganglia, cerebellum and brainstem GIq, and noise, respectively. These three 4-point grading scales are summarized in Table 1.

Based on PET images, five other dichotomic $(\mathrm{Y} / \mathrm{N})$ qualitative scores were also assessed: presence of hypermetabolic metastases, hypometabolic metastases (defined as hypometabolic compared to brain cortex and basal ganglia), edema, mass effect, and blurring of the target lesion.

\section{Quantitative Imaging Analysis}

Quantitative analyses were performed by the same two independent blinded readers (D. P. and V. L). The maximum standardized uptake value (SUVmax) of each brain metastasis was recorded using a standard volume of interest (VOI) tool on PET/CT images. Herewith, the VOI was automatically propagated to cover exactly the same volume in all eight different reconstruction datasets to ensure consistency of the area selected among different reconstructions in order to extract semiquantitative parameters. Moreover, background mean standardized uptake value (SUVmean) was assessed segmented manually using a banana-shaped VOI in normally appearing contralateral frontal and parietal brain parenchyma, including WM and GM. Based on these measurements, a target-to-background ratio (TBR) was calculated for each brain metastasis, defined as metastasis' SUVmax/background SUVmean.

We also defined a contrast recovery (CR) ratio comparing the target-to-background ratio of a BSREM reconstruction (numerator) to the target-to-background ratio of the reference OSEM reconstruction (denominator):

$C R=\frac{\left(\frac{\text { SUVmax }_{\text {SUVmean }}}{\text { Skgnd }}\right)-1}{\left(\frac{\text { SUVmax }_{\text {ref }}}{\text { SUVmean }_{\text {bkgnd }}}\right)-1}$

Table 1 Image grading scores where SUVmax is obtained from the brain metastasis and SUVmean from the background ("bkgnd"). Finally, "ref" indicates the reference reconstruction, as previously explained by ter Voert er al. [31]. The CR ratio is intended to highlight the advantage or disadvantage of each reconstruction compared to the ones used in clinical routine. In our study, we used both OSEM and BSREM 400 as reference reconstructions.

\section{Statistical Analyses}

Categorical variables are expressed as proportions, and continuous variables are presented as mean \pm standard deviation (SD) or median (range), depending on the distribution of values. For qualitative parameters, we compared the eight reconstruction techniques with respect to the qualitative image ratings (GIq, noise score, overall lesions detectability) using the non-parametric Friedman test for multiple samples. For quantitative parameters, brain metastases' SUVmax, background SUVmean, TBR, and CR were compared among all reconstruction techniques, using analysis of variances for repeated measures, with post-hoc Bonferroni corrections to adjust for multiple comparisons.

Further, differences in brain metastases' SUVmax were compared between patients with-low $(<25, n=21)$ and high ( $>25, n=19) \mathrm{BMI},-$ low $(\leq 2.0 \mathrm{MBq} / \mathrm{kg}$ body weight; $n=12)$ and a high ( $>2.0 \mathrm{MBq} / \mathrm{kg}$ body weight; $n=28)$ administered 18F-FDG activity,--low $(<5.5 \mathrm{mmol} / \mathrm{l} ; n=18)$ and high ( $>5.5 \mathrm{mmol} / \mathrm{l} ; n=22)$ glucose levels, and-small $(<1.5 \mathrm{~cm}$ in longest diameter; $n=29)$ and large $(>1.5 \mathrm{~cm}$ in longest diameter; $n=11$ ) metastases using Mann-Whitney $U$ test.

Cohen's kappa coefficient $(k)$ was used to measure interrater agreement for qualitative scores, such as GIq, noise score, overall lesions detectability and the presence of hypermetabolic metastases, hypometabolic metastases, edema, mass effect, and blurring of the target lesion. Cohen's kappa was interpreted as follows: $\leq 0$ no agreement, 0.01-0.20 as none to slight, $0.21-0.40$ fair, $0.41-0.60$ moderate, $0.61-0.80$ substantial, and $0.81-1.00$ almost perfect agreement [55]. Statistical significance was considered for

\section{Table 1 Inage grading scores}

\begin{tabular}{llll}
\hline Category & $\begin{array}{l}\text { General image quality (based on GM/ } \\
\text { WM score) }\end{array}$ & Noise score & $\begin{array}{l}\text { Lesion } \\
\text { detectability } \\
\text { score }\end{array}$ \\
\hline 1 & Poor (inadequate image with blurring) & Slight (almost none) & Poor \\
2 & Fair (diagnostically relevant image blurring) & Fair (diagnostic irrelevant) & Average \\
3 & Good (diagnostic irrelevant image blurring) & Moderate (diagnostic relevant) & Good \\
4 & Excellent (almost no blurring) & Severe (marked) & Very good \\
\hline
\end{tabular}

$G M$ grey matter, $W M$ white matter 
$p<0.05$. Statistical analyses were performed using IBM SPSS version 26.0 (IBM, Armonk, NY, USA) [56].

\section{Results}

Of the 40 patients included in this study (25 M, 15F), $77.5 \%$ underwent PET/CT for staging and $22.5 \%$ for restaging. The majority of patients were already deemed stage IV (70\%), based on CT imaging performed before the PET/CT scan (thereof $8 / 28 \mathrm{M} 1 \mathrm{a}, 6 / 28 \mathrm{M} 1 \mathrm{~b}, 14 / 28 \mathrm{M} 1 \mathrm{c}$ ). Of the 40 patients, $5 / 40(12.5 \%)$ had SCLC and 35/40 (87.5\%) had NSCLC (4/40 (10.0\%) squamous cell carcinoma, 24/40 (60.0\%) adenocarcinoma, and 7/40 (17.5\%) poorly differentiated carcinoma).

Based on the reference reconstructions for digital PET/ CT scan $\left(\right.$ BSREM $\left._{400}\right)$, clinical PET/CT reports (not further analyzed as part of this study) for brain metastases were positive, doubtful and negative in 16/40 (40\%), 2/40 (5\%), and $22(55 \%)$ patients, respectively.

The overall number of BM detected retrospectively with PET/CT in our study were $67,69,70,65,59,53,48$, and 49 at BSREM $_{100}, \mathrm{BSREM}_{200}, \mathrm{BSREM}_{300}, \mathrm{BSREM}_{400}$, BSREM $_{500}$, BSREM $_{600}$, BSREM $_{700}$, and OSEM, respectively. The mean number of BM detected was $1.63 \pm 1.48$ (median $=1 ; 0-7$ ) per patient at clinical 18F-FDG PET/ CT with BSREM $_{400}$ reconstruction versus $4.42 \pm 5.93$ (median $=2 ; 1-30$; total 177) at MRI. BSREM 300 reconstruction showed the highest mean number of BM detected per patient $(1.75 \pm 1.46$; median $=1 ; 1-7)$. Patient and tumor characteristics are listed in Table 2.

\section{Qualitative Image Results}

The results of the subjective image assessment regarding general image quality (GIq) score, the noise score, and the overall lesion detectability are given in Table 3 . The results regarding the other five dichotomic qualitative scores (hypermetabolic metastases, hypometabolic metastases, edema, mass effect, and blurring of the target lesion) and the ones with regard to GIq and noise score of the four analyzed brain structures (neocortex, basal ganglia, cerebellum, and brainstem) are reported in supplemental table $\mathrm{S} 1$ and supplemental table $\mathrm{S} 2$, respectively.

General image quality, noise score, and overall lesions detectability were rated significantly different among all reconstruction algorithms at Friedman test for multiple samples ( $p<0.001$ for both readers).

The median quality score was by trend higher for the BSREM reconstruction with lower $\beta$-values, with a similar decreasing trend of qualitative score rating for both readers. In particular, the median quality score for BSREM ${ }_{100}$
Table 2 Demographic data of study subjects $(n=40)$

\begin{tabular}{|c|c|}
\hline \multicolumn{2}{|l|}{ Patient and tumor characteristics } \\
\hline $\begin{array}{l}\text { PET/CT scan, } n(\%) \\
\quad \text { Staging } \\
\text { Restaging }\end{array}$ & $\begin{array}{l}35(87.5 \%) \\
5(12.5 \%)\end{array}$ \\
\hline $\begin{array}{l}\text { Gender, } n(\%) \\
\quad \text { Male } \\
\text { Female }\end{array}$ & $\begin{array}{l}25(62.5 \%) \\
15(37.5 \%)\end{array}$ \\
\hline Age (years), median (range) & $66.5(31-89)$ \\
\hline Activity injected (MBq), median (range) & $230.0(89-302)$ \\
\hline Uptake time (min), median (range) & $57.5(47-78)$ \\
\hline Blood glucose level (mmol/L), median (range) & $5.6(4.5-7.8)$ \\
\hline Weight (kg), median (range) & $75.5(47-111)$ \\
\hline Height $(\mathrm{cm})$, median (range) & $172.0(151-185)$ \\
\hline$B M I\left(\mathrm{~kg} / \mathrm{m}^{2}\right)$, median (range) & $24.7(18.4-37.5)$ \\
\hline \multicolumn{2}{|l|}{ Primary lung characteristics } \\
\hline $\begin{array}{l}\text { Histological type, } n(\%) \\
\text { Small-cell lung carcinoma (SCLC) } \\
\text { Non-small-cell lung carcinoma (NSCLC) } \\
\text { Squamous cell carcinoma } \\
\text { Adenocarcinoma } \\
\text { Poorl differentiated }\end{array}$ & $\begin{array}{l}5(12.5 \%) \\
35(87.5 \%) \\
4(10.0 \%) \\
24(60.0 \%) \\
7(17.5 \%)\end{array}$ \\
\hline $\begin{array}{l}\text { Location, } n(\%) \\
\text { Right lung } \\
\text { Left lung } \\
\text { Unknown }\end{array}$ & $\begin{array}{l}22(55.0 \%) \\
17(42.5 \%) \\
1(2.5 \%)\end{array}$ \\
\hline $\begin{array}{l}\text { Stage before }{ }^{18} F-F D G \text { PET/CT, } n(\%) \\
\quad I \\
I I A \\
\text { IIIB } \\
\text { IV } \\
\text { Unknown }\end{array}$ & $\begin{array}{l}1(2.5) \\
1(2.5) \\
1(2.5) \\
28(70.0) \\
9(22.5)\end{array}$ \\
\hline \multicolumn{2}{|l|}{ Brain metastasis characteristics } \\
\hline $\begin{array}{l}\text { Clinical PET/CT report on brain metastases } \\
\text { detection*, } n(\%) \\
\text { Positive } \\
\text { Doubtful } \\
\text { Negative }\end{array}$ & $\begin{array}{l}16(40.0 \%) \\
2(5.0 \%) \\
22(55.0 \%)\end{array}$ \\
\hline $\begin{array}{l}\text { Number of brain metastases per patient } \\
\text { detected with }{ }^{18} \mathrm{~F}-\mathrm{FDG} \text { PET/CT } \\
\text { mean } \pm \mathrm{DS} \text { (median, range) } \\
\text { BSREM }_{100} \\
\text { BSREM }_{200} \text { BSREM }_{300} \\
\text { BSREM }_{400} \\
\text { BSREM }_{500} \\
\text { BSREM }_{600} \\
\text { BSREM }_{700} \\
\text { OSEM }\end{array}$ & $\begin{array}{l}1.68 \pm 1.38(1 ; 1-7) \\
1.73 \pm 1.43(1 ; 1-7) \\
1.75 \pm 1.46(1 ; 1-7) \\
1.63 \pm 1.48(1 ; 0-7) \\
1.48 \pm 1.50(1 ; 0-7) \\
1.33 \pm 1.40(1 ; 0-7) \\
1.20 \pm 1.28(1 ; 0-6) \\
1.23 \pm 1.27(1 ; 0-6)\end{array}$ \\
\hline
\end{tabular}

\footnotetext{
* Based on findings detected on clinical PET with BSREM reconstruction with a $\beta$ value of 400 , which represents the clinical standard at our institution[33-36]

** Based on inter-reader agreement: readers identified cerebral metastases by reading both PET and CT images. In case of discrepancy of image rating, a final decision was made by consensus including a third reader, with 11 years of experience in nuclear medicine and radiology
} 
Table 3 Results of subjective PET image quality rating and PET parameters for different reconstruction algorithms

\begin{tabular}{|c|c|c|c|c|c|c|c|c|c|c|c|c|}
\hline \multirow[t]{3}{*}{ Reconstruction } & \multicolumn{4}{|c|}{ General image quality score } & \multicolumn{4}{|c|}{ Noise score } & \multicolumn{4}{|c|}{ Lesion detectability score } \\
\hline & \multicolumn{2}{|c|}{ Reader 1} & \multicolumn{2}{|c|}{ Reader 2} & \multicolumn{2}{|c|}{ Reader 1} & \multicolumn{2}{|c|}{ Reader 2} & \multicolumn{2}{|c|}{ Reader 1} & \multicolumn{2}{|c|}{ Reader 2} \\
\hline & Mean & SD & Mean & SD & Mean & SD & Mean & SD & Mean & SD & Mean & SD \\
\hline BSREM $_{100}$ & 2.14 & 1.13 & 3.63 & 0.59 & 3.73 & 0.67 & 3.9 & 0.49 & 2.60 & 1.08 & 2.60 & 1.37 \\
\hline BSREM $_{200}$ & 2.59 & 1.10 & 3.68 & 0.50 & 3.03 & 0.76 & 3.4 & 0.83 & 2.83 & 1.17 & 2.68 & 1.32 \\
\hline BSREM $_{300}$ & 2.58 & 1.04 & 3.15 & 0.72 & 2.03 & 0.88 & 2.75 & 0.94 & 2.78 & 1.23 & 2.68 & 1.36 \\
\hline BSREM $_{400}$ & 2.39 & 1.02 & 2.90 & 0.64 & 1.44 & 0.72 & 2.00 & 1.07 & 2.7 & 1.28 & 2.60 & 1.35 \\
\hline BSREM $_{500}$ & 2.24 & 1.01 & 2.46 & 0.77 & 1.13 & 0.42 & 1.40 & 0.80 & 2.56 & 1.25 & 2.35 & 1.38 \\
\hline BSREM $_{600}$ & 1.96 & 0.83 & 2.13 & 0.76 & 1.06 & 0.31 & 1.02 & 0.15 & 2.30 & 1.2 & 2.28 & 1.35 \\
\hline BSREM $_{700}$ & 1.63 & 0.70 & 1.91 & 0.71 & 1.01 & 0.08 & 1.02 & 0.15 & 2.08 & 1.16 & 2.25 & 1.31 \\
\hline OSEM & 1.58 & 0.74 & 1.94 & 0.84 & 1.09 & 0.41 & 1.23 & 0.72 & 2.30 & 1.11 & 2.28 & 1.33 \\
\hline \multirow[t]{3}{*}{ Reconstruction } & \multicolumn{4}{|c|}{ SUVmax } & \multicolumn{4}{|c|}{ SUVmean } & \multicolumn{4}{|l|}{ TBR } \\
\hline & \multicolumn{2}{|c|}{ Reader 1} & \multicolumn{2}{|c|}{ Reader 2} & \multicolumn{2}{|c|}{ Reader 2} & \multicolumn{2}{|c|}{ Reader 2} & \multicolumn{2}{|c|}{ Reader 1} & \multicolumn{2}{|c|}{ Reader 2} \\
\hline & Mean & SD & Mean & SD & Mean & SD & Mean & SD & Mean & SD & Mean & SD \\
\hline$\overline{\text { BSREM }}_{100}$ & 13.47 & 6.69 & 13.85 & 6.62 & 6.44 & 2.21 & 5.85 & 1.63 & 2.19 & 1.05 & 2.42 & 1.08 \\
\hline BSREM $_{200}$ & 11.68 & 5.95 & 11.91 & 6.02 & 6.11 & 1.96 & 5.81 & 1.60 & 1.96 & 0.93 & 2.08 & 0.95 \\
\hline BSREM $_{300}$ & 10.75 & 5.37 & 10.95 & 5.48 & 6.03 & 1.91 & 5.81 & 1.60 & 1.82 & 0.83 & 1.91 & 0.85 \\
\hline BSREM $_{400}$ & 10.15 & 5.08 & 10.32 & 5.02 & 5.8 & 1.71 & 5.81 & 1.60 & 1.76 & 0.76 & 1.79 & 0.77 \\
\hline BSREM $_{500}$ & 10.01 & 4.83 & 9.87 & 4.63 & 5.77 & 1.70 & 5.90 & 1.65 & 1.75 & 0.72 & 1.68 & 0.67 \\
\hline BSREM $_{600}$ & 9.95 & 4.45 & 9.53 & 4.31 & 5.74 & 1.63 & 5.97 & 1.72 & 1.75 & 0.69 & 1.61 & 0.62 \\
\hline BSREM $_{700}$ & 9.84 & 4.21 & 9.26 & 4.04 & 5.78 & 1.60 & 5.94 & 1.70 & 1.72 & 0.67 & 1.58 & 0.61 \\
\hline OSEM & 9.34 & 3.44 & 9.11 & 3.6 & 5.67 & 1.80 & 5.85 & 1.58 & 1.68 & 0.59 & 1.57 & 0.57 \\
\hline
\end{tabular}

BSREM block sequential regularized maximization, OSEM ordered subset expectation maximization, TBR target-to-background ratio

was $2.14 \pm 1.13$ (reader 1) and $3.63 \pm 0.59$ (reader 2), for BSREM $_{200} 2.59 \pm 1.10$ (reader 1) and $3.68 \pm 0.50$ (reader 2), and for BSREM ${ }_{300} 2.58 \pm 1.04$ (reader 1) and $3.68 \pm 0.50$ (reader 2). The most evident inter-reader discrepancy was due to a higher average evaluation by reader 2 compared to reader 1 , which is accentuated for the $\mathrm{BSREM}_{100}$ reconstruction (Table 3). This data is reflected in the low agreement of the two readers for this particular score with a weighted $k<0.05$ for the majority of reconstructions, with the exception of $\mathrm{BSREM}_{200}(k=0.268$ and $95 \%$ confidence interval (CI) $0.09-0.44)$ and OSEM $(k=0.306$ and $95 \%$ CI 0.12-0.48) (supplemental table S3).

In contrast, inter-reader agreement was high for both noise and lesion detectability, both decreasing with higher $\beta$-value and OSEM. OSEM reconstruction presented a similar performance, as BSREM ${ }_{700}$. The overall detectability of brain metastases was higher for BSREM $_{200}$ and BSREM $_{300}$ for both readers compared to all other reconstructions. Owing to deterioration of image quality by noise, BSREM $_{100}$ accuracy was lower. In the analysis of brain structures, both readers assigned a lower GIq to cerebellum and brainstem compared to neocortex and basal ganglia with a similar trend, even if GIq score by reader 2 was generally higher compared to reader 1, as shown in Fig. 1.

Finally, with regard to the dichotomic scores, agreements were surprisingly high for photopenic brain metasta- ses, which turned out to be highly detectable regardless of the reconstruction algorithm used (supplemental Fig. 2), and for metastases' blurring, which was slightly higher with higher $\beta$-value and OSEM for reader 2 .

\section{Quantitative Image Results}

Mean values of PET parameters for both readers are given in Table 3; mean differences of PET parameters and $p$ values of pairwise comparisons using different reconstruction algorithms are given in supplemental table S4.

Apart from pairwise comparisons (supplemental table S4), only BSREM ${ }_{100}$ was significantly different from $\mathrm{BSREM}_{500}(p=0.017), \mathrm{BSREM}_{600}(p=0.006), \mathrm{BSREM}_{700}$ $(p=0.002)$, and OSEM $(p=0.002)$ for metastases' SUVmax and from $\operatorname{BSREM}_{400}(p=0.014), \operatorname{BSREM}_{500}(p=0.001)$, $\operatorname{BSREM}_{600}(p=0.0001), \operatorname{BSREM}_{700}(p=0.0001)$, and $\operatorname{OSEM}(p=0.0001)$ for TBR. The estimated marginal means presented in Fig. 2 reveal a significant and progressive reduction of both PET parameters with increasing $\beta$-value,

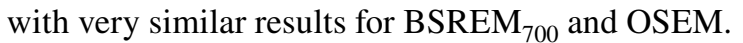

These data are corroborated also by the results of the contrast recovery $(\mathrm{CR})$ ratio comparing PET parameters of a given reconstruction with the reference reconstruction (supplemental fig. S3). The box plot representation of the CR ratio with $\mathrm{BSREM}_{400}$ as reference reconstruc- 


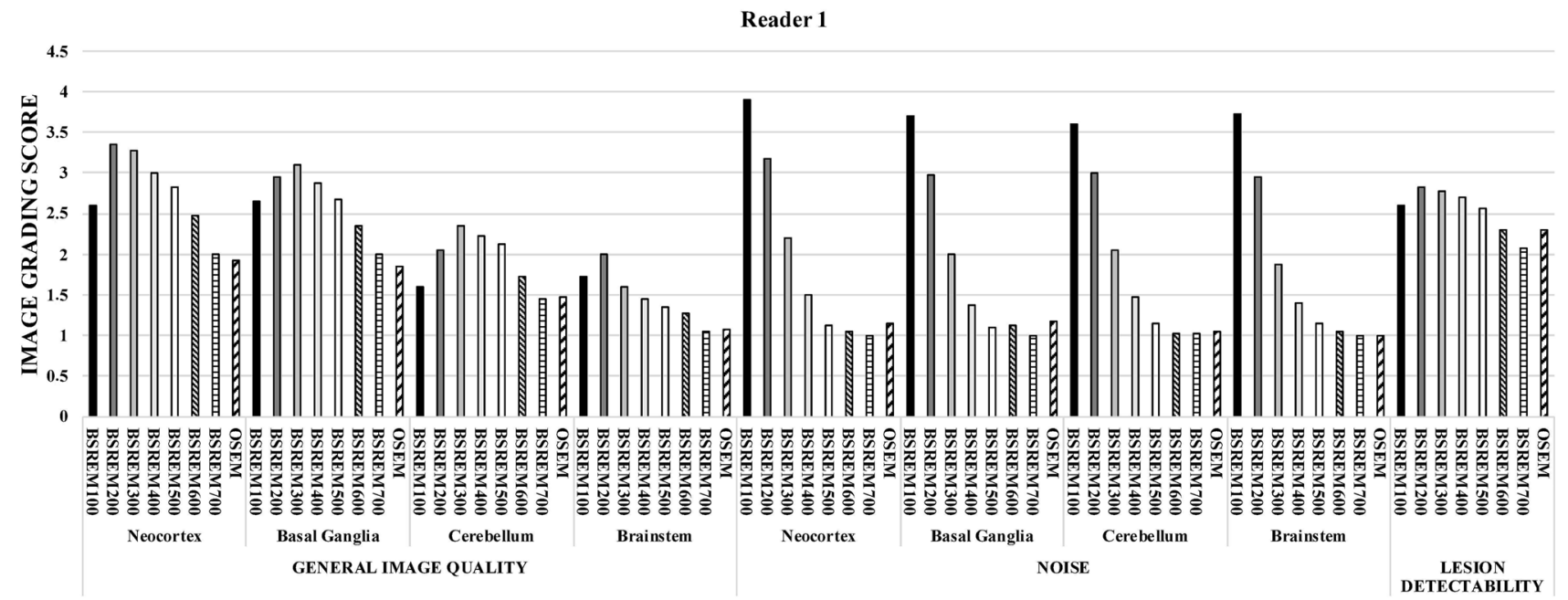

Reader 2

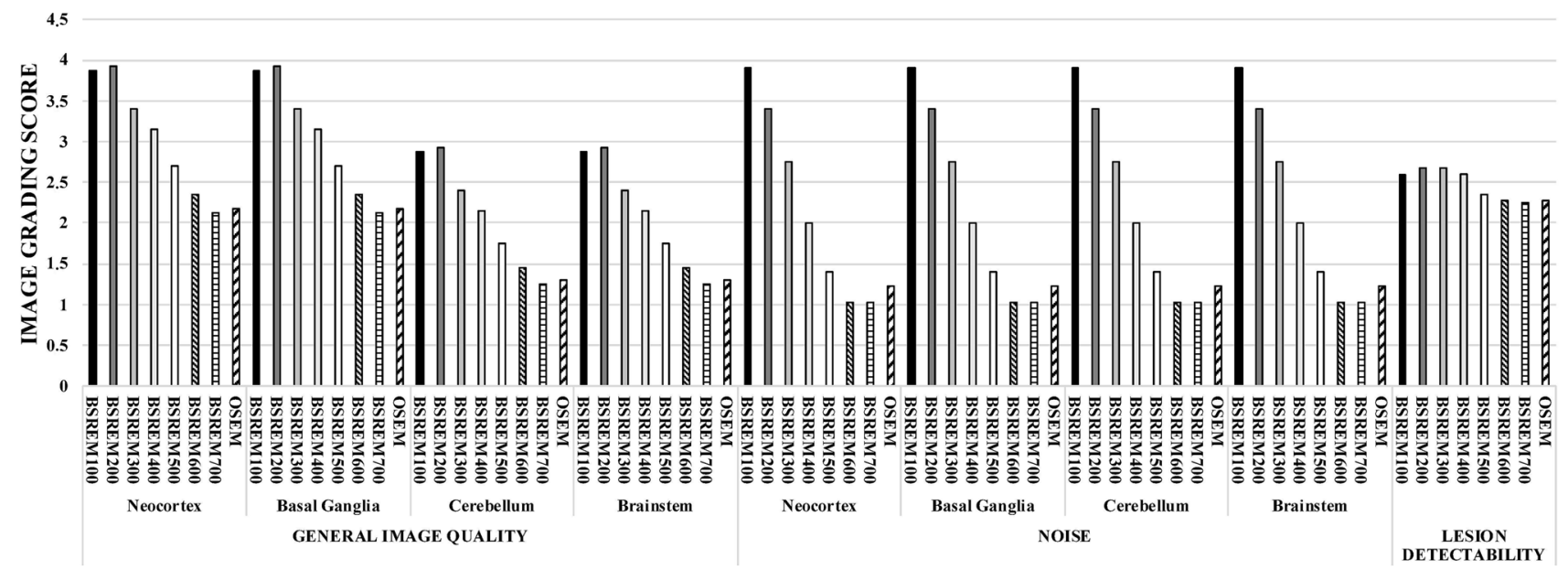

Fig. 1 Visual scoring of general image quality and noise of neocortex, basal ganglia, cerebellum, and brainstem, respectively, and overall lesion detectability evaluated by the two readers per arbitrary image grading score, as described in Table 2

tion reveals an added value of $\mathrm{BSREM}_{100}, \mathrm{BSREM}_{200}$, and BSREM $_{300}$ in comparison to the other reconstruction algorithms, leading to a better definition of tiny lesions in BSREM with lower $\beta$-values, as shown in Fig. 3.

Finally, the Mann-Whitney $U$ test for differences in brain metastases' SUVmax showed no significant impact of lesion size on SUVmax, while BMI $>25$ and MBq/ $\mathrm{kg}<2.0$ impacted SUVmax for BSREM reconstruction with higher $\beta$-value and for OSEM, but without significance, except for BMI $>25$ in OSEM (supplemental table S5).

\section{Discussion}

To the best of our knowledge, this study is the first one that sought to analyze different PET reconstruction algorithms for the assessment of brain metastases in patients with lung cancer, using a latest-generation silicon-based digital $\mathrm{PET} / \mathrm{CT}$ scanner.

The major findings of our study are as follows: (1) BSREM with lower $\beta$-values (namely, $\mathrm{BSREM}_{100}, \mathrm{BSREM}_{200}$, and BSREM $_{300}$ ) is better suited for the detection of BM than BSREM with higher $\beta$-values or OSEM; (2) BSREM reconstruction leads to a significant increase of SUVmax and TBR, being most prominent with lower $\beta$-values; (3) despite that, BSREM $_{100}$ sensitivity is affected by the presence of high noise levels compared to other reconstructions; (4) PET/CT sensitivity for BM detection is not affected by brain lesion size, regardless of the reconstruction algorithm used; (5) BMI $>25$ and $\mathrm{MBq} / \mathrm{kg}<2.0$ could reduce the sensitivity of both high $\beta$-value BSREM and OSEM for BM detection (but future prospective data are needed to validate this finding), and (6) both readers agreed that $\mathrm{BSREM}_{200-300}$ leads to a significant increase in BM detectability compared to BSREM $_{400}$, which is normally used in clinical practice for whole-body exams. 
Fig. 2 Profile plots of the estimated marginal means of PET parameters (brain metastases' SUVmax, background SUVmean, and TBR) derived by pairwise comparisons of different reconstruction algorithms
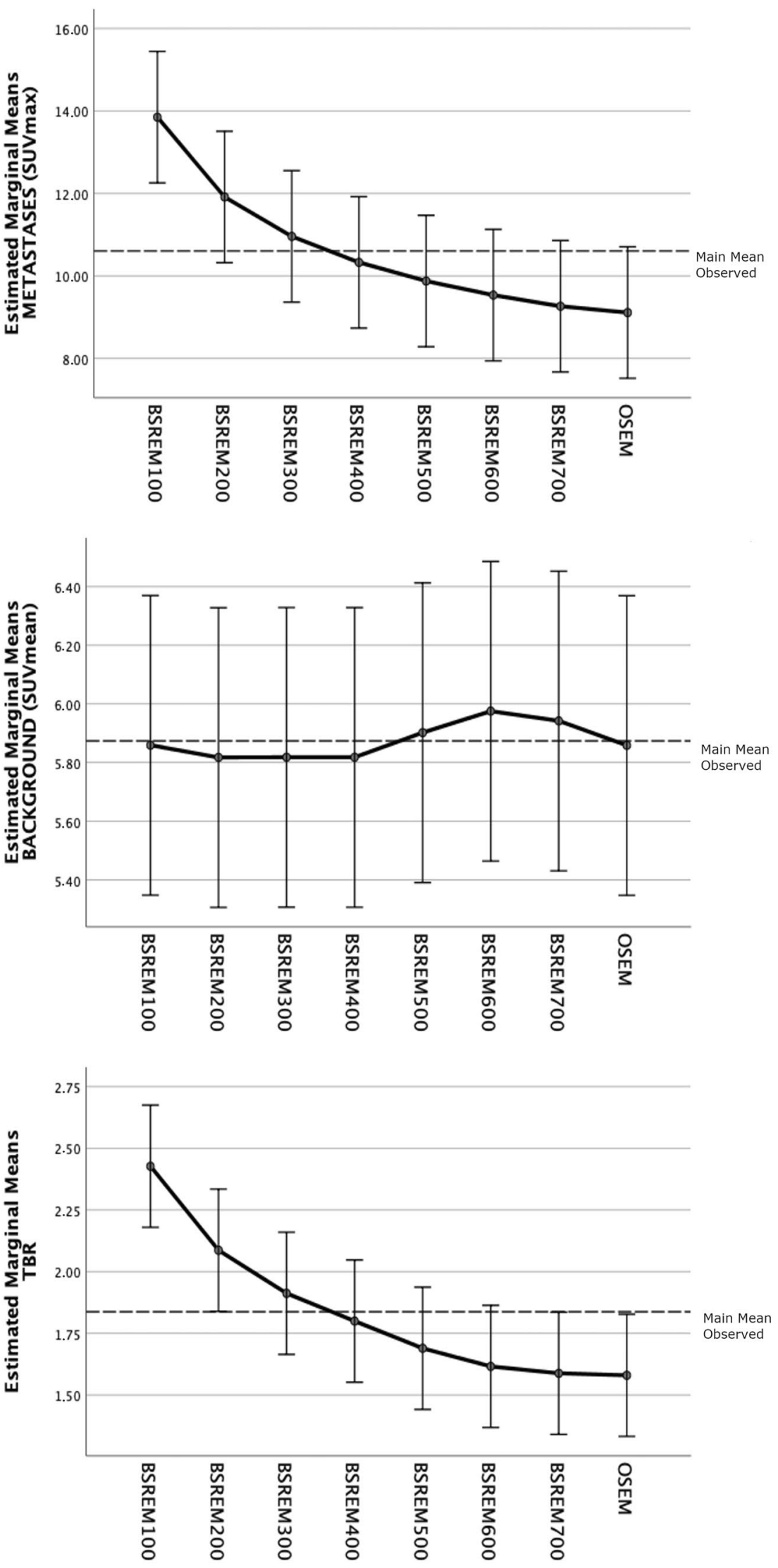


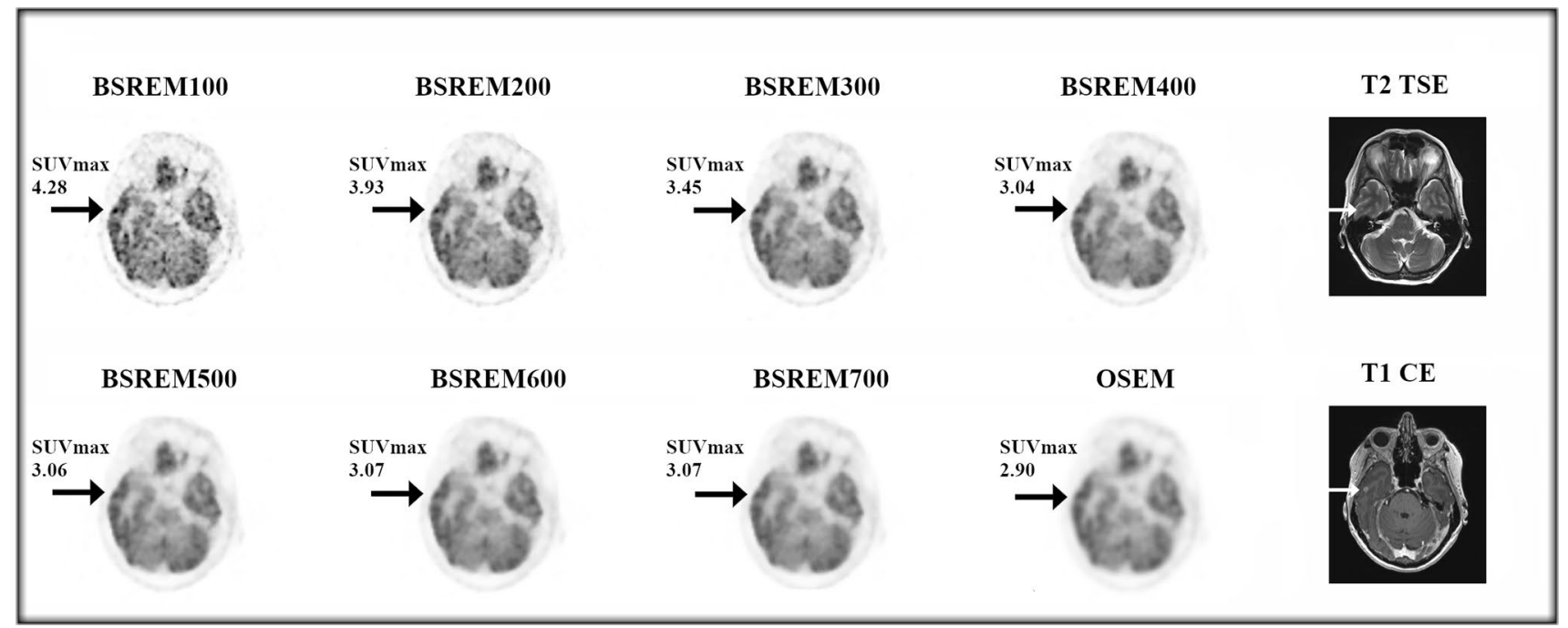

Fig. 3 Exemplary case of a 62-year-old patient undergoing restaging ${ }^{18}$ F-FDG PET/CT performed for an adenocarcinoma of the left lung with lymph node metastases and brain metastases (T1c Nx M1b, stage IVB). The MR images performed 20 days after PET/CT

Several studies have previously compared different PET reconstruction algorithms with regard to image quality and quantitative parameters in whole-body oncological subsets, but none of them focused on 18F-FDG brain PET/CT images in oncological subsets. Notably, all studies analyzing the impact of different reconstructions in 18F-FDG PET/CT whole-body exams [27, 34-36, 57] reported an ideal $\beta$-value between 400 and 500 for BSREM reconstructions. Two other studies focusing on the evaluation of prostate cancer with 68 Ga-PSMA-11 [31, 58] reported higher ideal $\beta$-values for this high energy positron emitter, slightly higher with ter Voert et al. (PET/MR study, BSREM B00-600 ) and considerably higher with Lindström et al. (PET/CT study, BSREM ${ }_{900}$ ), which, however, might be explained by the known differences in $\beta$-value characteristics between PET/MR and PET/CT. In another study, Lindström et al. [59] identified tracer-specific ranges of $\beta$-values for BSREM reconstruction according to the different biodistribution of the tracers, different background uptake, and different positron range of 18F-FDG, 11C-acetate, and $68 \mathrm{Ga}-$ DOTATOC in tissue. According to their findings, $18 \mathrm{~F}-\mathrm{FDG}$ requires lower $\beta$-values without compromising the image quality in terms of increased noise, while 68 Ga-DOTATOC images benefit from higher $\beta$-values.

These data corroborate the impossibility to identify one single optimal $\beta$-value suitable for all radiopharmaceuticals and all scan indications. To the best of our knowledge, to date only Shkumat et al. [60] have quantified the diagnostic performance of OSEM and BSREM ( $\beta$-values 200 , 350 , and 500) reconstructions in brain 18F-FDG PET/CT images of twenty-five pediatric epilepsy patients on a digital silicon photomultiplier system, stratified also by different acquisition times (45 s, $90 \mathrm{~s}, 180 \mathrm{~s}, 300 \mathrm{~s})$ in order to shows a tiny brain metastasis in the right-sided temporal lobe (white arrows), which was detected by both readers only on BSREM B $_{100}$ and BSREM $_{200}$ reconstructions and is faintly present also on BSREM 300

simulate reduced count density. They report that pediatric brain 18F-FDG PET/CT images remain diagnostic with a reduction of count density by $40 \%$ when using a $\beta$-value of 350-500. Moreover, they also identified a reduction of image quality of cortex, basal ganglia, and thalamus when applying lower $\beta$-values due to increasing noise, particularly in the thalamus. In their study, the highest spatial resolution was reported for a $\beta$-value of 200-300.

Results of our study are in line with these findings,

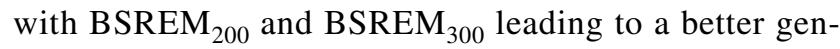
eral image quality being counterbalanced by acceptable background noise, in contrast to BSREM $_{100}$. In addition, based on the subjective evaluation of the two readers, spatial resolution seemed to be higher when applying BSREM100-300, whereas higher $\beta$-values resulted in a loss of gray matter/white matter contrast, especially for smaller structures, such as basal ganglia and brainstem. A quantitative assessment of spatial resolution should be performed to validate this hypothesis.

These findings may not only be of significance in the oncological field for BM detection, but also in neurodegenerative disorders. Recently, Lindström et al. [38] have evaluated how different $\beta$-values (BSREM; TOF, PSF, $\beta$-value 75-300) can affect quantitative measures and software-aided assessment of pathologies in patients with neurodegenerative diseases compared to cognitively normal controls, in both $18 \mathrm{~F}$-flutemetamol and 18F-FDG PET/CT imaging. They conclude that BSREM image reconstructions should be used with caution when a normal's database was collected based on images acquired with OSEM reconstruction. Moreover, they reported that 
TOF, PSF, and BSREM either increased or decreased the relative uptake difference to the normal's database within the software, depending on the radiotracer and chosen reference area.

While the right choice between $\mathrm{BSREM}_{200}$ and BSREM $_{300}$ remains arbitrary and somewhat subjective, it seems clearer that intermediate-high $\beta$-value (600-700) are not suitable for a correct evaluation of brain 18F-FDG PET/ CT images, both qualitatively and quantitatively with regard to PET parameters. BSREM ${ }_{600}$ and BSREM ${ }_{700}$ show a considerable reduction in the number of detected metastases (mean $1.33 \pm 1.40$ and $1.20 \pm 1.28$, respectively), compared to $\mathrm{BSREM}_{300}$ (mean $1.75 \pm 1.46$ ). This comes along with a significant drop of the measured semi-quantitative PET parameter values with $\mathrm{BSREM}_{600}$ and $\mathrm{BSREM}_{700}$, which were within the range of those measured in OSEM. These data are in line with previous findings by Caribé et al. [61], who already reported that BSREM $_{750}$ has a resolution comparable to OSEM, but with a background noise level reduced by a factor of 4 (coefficient of variation, COV), overall reducing the detectability of small lesions in BSREM $_{750}$ compared to lower $\beta$-value BSREM, as shown in the example in supplemental fig. S4.

Literature data suggests a correlation between lesion dimension and PET/CT detectability [51], which was, however, not observed in our study. One explanation might be the heterogeneity of biological behavior of BM, leading to varying FDG patterns (hypermetabolic, isometabolic, and hypometabolic, the latter indicating prominent perifocal edema), as already mentioned by Hjorthaug et al. [21]. Interestingly, our results suggest a possible association of BMI $>25$ and $\mathrm{MBq} / \mathrm{kg}<2.0$ with lower sensitivity of both BSREM with higher $\beta$-values and OSEM, although without statistical significance. Recently, Messerli et al. [32] highlighted how the optimal $\beta$-value choice in whole-body $18 \mathrm{~F}$ FDG PET/CT could depend on the administered activity $(\mathrm{MBq} / \mathrm{kg}<$ or $>2.0)$. Future prospective data with a larger cohort of patients are needed to validate these findings.

To conclude, despite all the known limitations of 18FFDG PET/CT images in this field, the use of BSREM reconstruction with $\beta$-values of 200-300 could increase the sensitivity of $18 \mathrm{~F}-\mathrm{FDG}$ PET/CT images to detect brain metastases.

We acknowledge that MR imaging undoubtedly represents the reference standard for the cerebral staging of advanced lung cancer patients. As mentioned, a staging MRI is considered mandatory for NSCLC patients stage II or higher [62-64]. However, data from more than 457,000 NSCLC patients reported by Wagar et al. [65] proofs that while the prevalence of $\mathrm{BM}$ at initial presentation doubles from stage I to stage II (from 2.3 to $4.6 \%$ ) - only $74 \%$ of stage II patients actually undergo MR imaging (27\% of stage I patients). Even in stages II and IV (BM prevalence 10.8\% and $12.1 \%$, respectively), a brain MRI was available only in $92 \%$ and $88 \%$ of patients, respectively.

Furthermore, if no BM where detected at initial staging, and in the absence of neurological symptoms, a regular MRI surveillance of the brain is oftentimes not performed, also in subjects undergoing regular follow-up PET/CT scans for whole-body surveillance. In these subjects, adequate PET imaging of the brain - as part of the whole-body exam - might be critical. Notably, lung cancer restaging represents one of the most common PET indications, owing to the general high incidence of lung cancer and owing to available reimbursement for this disease in most countries with PET reimbursement schemes. Also, some patients cannot undergo MRI examinations owing to contraindications. In these subjects, adequate PET imaging of the brain might assume a more prominent role, possibly in conjunction with contrast-enhanced (ce)-CT, which was however not analyzed in our study.

Hence, the detection of BM by 18F-FDG PET/CT might have a positive impact on clinical management and patient outcome not only in advanced lung cancer patients, but also in all other solid tumors often metastatic to the brain (breast cancer, melanoma, RCC, and CRC), and in which brain MRI is prescribed only in case of high suspicion and/or presence of neurological symptoms.

Our study is not exempt from limitations. First, although readers were blinded to the type of reconstruction used, an experienced reader may recognize the actual algorithm used based on the reconstructed images. Second, despite

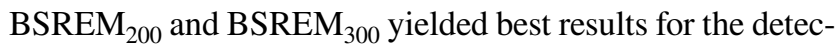
tion of brain metastases, a statistically significant difference in the number of brain metastases detected among the different reconstruction types was not found. The number of patients in this single center study is comparably small, and therefore, conclusions drawn from the present analysis await further proof in larger (and ideally multicentric) observations. Future studies are also warranted to assess the impact of BSREM depending on different primary cancer origin, different metabolic behavior of BM, and different brain regions involved for a better stratification of diagnostic accuracy, clinical management, and patient outcome. Since a detailed analysis of OSEM was not the thrust of our study, OSEM was not optimized, but clinically standard OSEM served as a reference only.

\section{Conclusion}

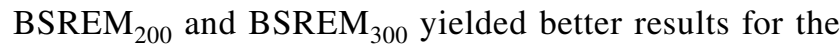
detection of brain metastases, being superior to BSREM $_{400}$ and OSEM, which are normally used in clinical practice for whole-body exams. With BSREM ${ }_{200}$ and BSREM $_{300}$, 
higher SUVmax, TBR, and an acceptable background noise translate into higher image quality and tumor conspicuity. In clinical routine, it might be useful to separately reconstruct and analyze such brain PET images in whole-body exams for the staging and restaging of lung cancer patients, particularly in the absence of synchronous brain MR imaging.

Supplementary Information The online version contains supplementary material available at https://doi.org/10.1007/s10278-021-00570-y.

Acknowledgements We would like to express our gratitude to the anonymous patients on whom this work is based and the staff of the Department of Nuclear Medicine for their excellent technical support.

Author Contribution MH designed the study; VL and DP reviewed the PET/CT scans and collected the data; VL conducted the statistical analysis on PET/CT scans; VL and MH wrote the manuscript. All authors discussed the results and commented on the manuscript. All authors reviewed the final manuscript.

Funding Open access funding provided by University of Zurich. The University Hospital Zurich holds a research agreement with GE Healthcare (unrelated to the current study). This study was supported by a specific IIS grant from GE Healthcare.

Availability of Data and Materials The datasets used and/or analyzed during the current study are available from the corresponding author on reasonable request.

\section{Declarations}

Ethical Approval All procedures performed in studies involving human participants were in accordance with ethical standards of the institutional and/or national committee and with the 1964 Helsinki declaration and its later amendments or comparable ethical standards.

Consent to Participate Informed consent was obtained from all individual participants included in the study.

Consent for Publication Additional informed consent was obtained from all individual participants for whom identifying information is included in this article.

Conflict of Interest $\mathrm{MH}$ is a recipient of grants and speaker's fees from GE Healthcare, grants for translational and clinical cardiac and oncological research from the Alfred and Annemarie von Sick legacy, and grants from the Artificial Intelligence in oncological Imaging Network by the University of Zurich.

Open Access This article is licensed under a Creative Commons Attribution 4.0 International License, which permits use, sharing, adaptation, distribution and reproduction in any medium or format, as long as you give appropriate credit to the original author(s) and the source, provide a link to the Creative Commons licence, and indicate if changes were made. The images or other third party material in this article are included in the article's Creative Commons licence, unless indicated otherwise in a credit line to the material. If material is not included in the article's Creative Commons licence and your intended use is not permitted by statutory regulation or exceeds the permitted use, you will need to obtain permission directly from the copyright holder. To view a copy of this licence, visit http://creativecommons.org/licenses/by/4.0/.

\section{References}

1. Achrol AS, Rennert RC, Anders C, et al. Brain metastases [Internet]. Nat. Rev. Dis. Prim. Nature Publishing Group; 2019 [cited 2020]. Available from: https://pubmed.ncbi.nlm.nih.gov/ $30655533 /$

2. Lee H, Jeong SH, Jeong BH, et al. Incidence of brain metastasis at the initial diagnosis of lung squamous cell carcinoma on the basis of stage, excluding brain metastasis. J Thorac Oncol [Internet]. Lippincott Williams and Wilkins; 2016 [cited 2020];11:426-431. Available from: https://pubmed.ncbi.nlm. nih.gov/26746367/

3. Mamon HJ, Yeap BY, Jänne PA, et al. High risk of brain metastases in surgically staged IIIA non-small-cell lung cancer patients treated with surgery, chemotherapy, and radiation [Internet]. J. Clin. Oncol. J Clin Oncol; 2005 [cited 2020]. page 1530-1537. Available from: https://pubmed.ncbi.nlm.nih.gov/ $15735128 /$

4. N Duma R Santana-Davila Molina JR. Non-Small Cell Lung Cancer: Epidemiology, Screening, Diagnosis, and Treatment [Internet]. Mayo Clin. Proc. Elsevier Ltd; 2019 [cited, 2020 page 1623-1640 Available from: https://doi.org/10.1016/j.mayocp. 2019.01 .013

5. Hochstenbag M, Twijnstra A, Wilmink J, et al. Asymptomatic brain metastases (BM) in small cell lung cancer (SCLC): MRimaging is useful at initial diagnosis. J Neurooncol [Internet]. J Neurooncol; 2000 [cited 2020];48:243-248. Available from: https://pubmed.ncbi.nlm.nih.gov/11100822/

6. Patchell RA. Metastatic brain tumors. Neurol. Clin. W.B. Saunders; 1995. page 915-925.

7. Carolan H, Sun AY, Bezjak A, et al. Does the incidence and outcome of brain metastases in locally advanced non-small cell lung cancer justify prophylactic cranial irradiation or early detection? Lung Cancer [Internet]. Lung Cancer; 2005 [cited 2020]. page 109-115. Available from: https://pubmed.ncbi.nlm. nih.gov/15949596/

8. Andre F, Grunenwald D, Pujol JL, et al. Patterns of relapse of N2 nonsmall-cell lung carcinoma patients treated with preoperative chemotherapy: Should prophylactic cranial irradiation be reconsidered? Cancer [Internet]. John Wiley \& Sons, Ltd; 2001 [cited 2020];91:2394-2400. Available from: https://onlinelibrary.wiley. com/doi/https://doi.org/10.1002/1097-0142(20010615)91:12\% 3C2394::AID-CNCR1273\%3E3.0.CO;2-6

9. Gaspar LE, Chansky K, Albain KS, et al. Time from treatment to subsequent diagnosis of brain metastases in stage III nonsmall-cell lung cancer: A retrospective review by the Southwest Oncology Group [Internet]. J. Clin. Oncol. J Clin Oncol; 2005 [cited 2020]. page 2955-2961. Available from: https://pubmed. ncbi.nlm.nih.gov/15860851/

10. Schoenmaekers J, Hofman P, Bootsma G, et al. Screening for brain metastases in patients with stage III non-small-cell lung cancer, magnetic resonance imaging or computed tomography? A prospective study. Eur J Cancer [Internet]. Elsevier Ltd; 2019 [cited 2020];115:88-96. Available from: https://pubmed.ncbi. nlm.nih.gov/31129385/

11. Li Y, Jin G, Su D. Comparison of Gadolinium-enhanced MRI and 18FDG PET/PET-CT for the diagnosis of brain metastases in lung cancer patients: A meta-analysis of 5 prospective studies. Oncotarget [Internet]. Impact Journals, LLC; 2017 [cited 2020];8:35743-35749. Available from: https://pubmed.ncbi. nlm.nih.gov/28415747/

12. Planchard D, Popat S, Kerr K, et al. by the ESMO Guidelines Committee Metastatic non-small cell lung cancer: ESMO Clinical Practice Guidelines for diagnosis, treatment and follow-up $\dagger 29$ (suppl 4): iv192-iv237. Ann Oncol. 2018. 
13. Ettinger DS, Wood DE, Chair V, et al. Continue NCCN Guidelines Panel Disclosures NCCN Guidelines Version 1.2020 NonSmall Cell Lung Cancer. 2019.

14. Barrón-Barrón F, Guzmán-De Alba E, Alatorre-Alexander J, et al. Guía de Práctica Clínica Nacional para el manejo del cáncer de pulmón de células no pequeñas en estadios tempranos, localmente avanzados y metastásicos. Salud Publica Mex [Internet]. NLM (Medline); 2019 [cited 2020];61:359-414. Available from: https://pubmed.ncbi.nlm.nih.gov/31276353/

15. S Wang S Zimmermann K Parikh Current Diagnosis Management of Small-Cell Lung Cancer [Internet]. Mayo Clin. Proc. Elsevier Ltd; 2019 [cited, et al 2020 page 1599-1622 Available from: https://doi.org/10.1016/j.mayocp.2019.01.034

16. Kandathil A, Kay FU, Butt YM, et al. Role of FDG PET/CT in the eighth edition of TNM staging of non- Small cell lung cancer. Radiographics [Internet]. Radiological Society of North America Inc.; 2018 [cited 2020];38:2134-2149. Available from: www.ajronline.org

17. Grootjans W, De Geus-Oei LF, Troost EGC, et al. PET in the management of locally advanced and metastatic NSCLC [Internet]. Nat. Rev. Clin. Oncol. Nature Publishing Group; 2015 [cited 2020]. page 395-407. Available from: https://pubmed. ncbi.nlm.nih.gov/25917254/

18. PY Salaün R Abgral O Malard Good clinical practice recommendations for the use of PET, CT in oncology. Eur J Nucl Med Mol Imaging [Internet]. Springer, et al 2020 [cited 2020];47:2850 Available from: https://doi.org/10.1007/s00259-019-04553-8

19. S Fuchs N Grössmann M Ferch Evidence-based indications for the planning of PET or PET, CT capacities are needed [Internet]. Clin. Transl. Imaging. Springer-Verlag Italia s.r.1., 2019 [cited, et al 2020 page 65-81 Available from: https://doi.org/ 10.1007/s40336-019-00314-7

20. Bochev P, Klisarova A, Kaprelyan A, et al. Brain metastases detectability of routine whole body 18F-FDG PET and low dose CT scanning in 2502 asymptomatic patients with solid extracranial tumors. Hell J Nucl Med [Internet]. Hell J Nucl Med; 2012 [cited 2020];15. Available from: https://pubmed.ncbi.nlm.nih. gov/22741148/

21. Hjorthaug K, Højbjerg JA, Knap MM, et al. Accuracy of $18 \mathrm{~F}$ FDG PET-CT in triaging lung cancer patients with suspected brain metastases for MRI. Nucl Med Commun [Internet]. Lippincott Williams and Wilkins; 2015 [cited 2020];36:1084-1090. Available from: https://pubmed.ncbi.nlm.nih.gov/26302460/

22. Nia ES, Garland LL, Eshghi N, et al. Incidence of brain metastases on follow-up 18F-FDG PET/CT scans of non-small cell lung cancer patients: Should we include the brain? J Nucl Med Technol [Internet]. Society of Nuclear Medicine Inc.; 2017 [cited 2020];45:193-197. Available from: https://pubmed.ncbi.nlm. nih.gov/28705927/

23. Kung B, Auyong T, Tong C. Prevalence of Detecting Unknown Cerebral Metastases in Fluorodeoxyglucose Positron Emission Tomography/Computed Tomography and its Potential Clinical Impact. World J Nucl Med [Internet]. Medknow; 2014 [cited 2020];13:108. Available from: https://pubmed.ncbi.nlm.nih. gov/25191125/

24. Tasdemir B, Urakci Z, Dostbil Z, et al. Effectiveness of the addition of the brain region to the FDG-PET/CT imaging area in patients with suspected or diagnosed lung cancer. Radiol Medica [Internet]. Springer-Verlag Italia s.r.1.; 2016 [cited 2020];121:218-224. Available from: https://pubmed.ncbi.nlm. nih.gov/26541882/

25. Cheng JC, Matthews J, Sossi V, et al. Incorporating HYPR denoising within iterative PET reconstruction (HYPR-OSEM). Phys Med Biol [Internet]. Institute of Physics Publishing; 2017 [cited 2020];62:6666-6687. Available from: https://pubmed.ncbi.nlm. nih.gov/28644152/
26. Wolpert F, Weller M, Berghoff AS, et al. Diagnostic value of 18F-fluordesoxyglucose positron emission tomography for patients with brain metastasis from unknown primary site. Eur J Cancer [Internet]. Elsevier Ltd; 2018 [cited 2020];96:64-72. Available from: https://pubmed.ncbi.nlm.nih.gov/29677642/

27. Messerli M, Kotasidis F, Burger IA, et al. Impact of different image reconstructions on PET quantification in non-small cell lung cancer: A comparison of adenocarcinoma and squamous cell carcinoma. Br J Radiol [Internet]. British Institute of Radiology; 2019 [cited 2020];92. Available from: /pmc/articles/ PMC6540860/?report=abstract

28. Lois C, Jakoby BW, Long MJ, et al. An assessment of the impact of incorporating time-of-flight information into clinical PET/ CT imaging. J Nucl Med [Internet]. J Nucl Med; 2010 [cited 2020];51:237-245. Available from: https://pubmed.ncbi.nlm.nih. gov/20080882/

29. Salvadori J, Perrin M, Marie PY, et al. High-Resolution Brain 18F-FDG Images Provided by Fully Digital PET. Clin Nucl Med [Internet]. Lippincott Williams and Wilkins; 2019 [cited 2021];44:301-302. Available from: https://pubmed.ncbi.nlm.nih. gov/30789394/

30. Lantos J, Mittra ES, Levin CS, et al. Standard OSEM vs. regularized PET image reconstruction: qualitative and quantitative comparison using phantom data and various clinical radiopharmaceuticals. Am J Nucl Med Mol Imaging [Internet]. 2018 [cited 2020];8:110-118. Available from: http://www.ncbi.nlm.nih.gov/ pubmed/29755844

31. ter Voert EEGW, Muehlematter UJ, Delso G, et al. Quantitative performance and optimal regularization parameter in block sequential regularized expectation maximization reconstructions in clinical 68Ga-PSMA PET/MR. EJNMMI Res [Internet]. Springer Verlag; 2018 [cited 2020];8:70. Available from: http:// www.ncbi.nlm.nih.gov/pubmed/30054750

32. Messerli M, Stolzmann P, Egger-Sigg M, et al. Impact of a Bayesian penalized likelihood reconstruction algorithm on image quality in novel digital PET/CT: clinical implications for the assessment of lung tumors. EJNMMI Phys [Internet]. Springer International Publishing; 2018 [cited 2020];5:27. Available from: http://www. ncbi.nlm.nih.gov/pubmed/30255439

33. Bjöersdorff M, Oddstig J, Karindotter-Borgendahl N, et al. Impact of penalizing factor in a block-sequential regularized expectation maximization reconstruction algorithm for $18 \mathrm{~F}$-fluorocholine PETCT regarding image quality and interpretation. EJNMMI Phys [Internet]. Springer International Publishing; 2019 [cited 2020];6. Available from: https://pubmed.ncbi.nlm.nih.gov/30900064/

34. Trägårdh E, Minarik D, Almquist $\mathrm{H}$, et al. Impact of acquisition time and penalizing factor in a block-sequential regularized expectation maximization reconstruction algorithm on a Si-photomultiplier-based PET-CT system for 18F-FDG. EJNMMI Res [Internet]. Springer Verlag; 2019 [cited 2020];9:64. Available from: http://www.ncbi. nlm.nih.gov/pubmed/31342214

35. Lindström E, Sundin A, Trampal C, et al. Evaluation of penalizedlikelihood estimation reconstruction on a digital time-of-flight PET/CT scanner for 18 F-FDG whole-body examinations. J Nucl Med. Society of Nuclear Medicine Inc.; 2018;59:1152-1158.

36. Sah BR, Stolzmann P, Delso G, et al. Clinical evaluation of a block sequential regularized expectation maximization reconstruction algorithm in 18F-FDG PET/CT studies. Nucl Med Commun. Lippincott Williams and Wilkins; 2017;38:57-66.

37. Shkumat NA, Vali R, Shammas A. Clinical evaluation of reconstruction and acquisition time for pediatric 18F-FDG brain PET using digital PET/CT. Pediatr Radiol. Springer; 2020;50:966-972.

38. Lindström E, Oddstig J, Danfors T, et al. Image reconstruction methods affect software-aided assessment of pathologies of [18F] flutemetamol and [18F]FDG brain-PET examinations in patients with neurodegenerative diseases. NeuroImage Clin [Internet]. 
Elsevier Inc.; 2020 [cited 2020];28. Available from: https://pubmed.ncbi.nlm.nih.gov/32882645/

39. Vennart NJ, Bird N, Buscombe J, et al. Optimization of PET/CT image quality using the GE "Sharp IR" point-spread function reconstruction algorithm. Nucl Med Commun [Internet]. Lippincott Williams and Wilkins; 2017 [cited 2020];38:471-479. Available from: https:// pubmed.ncbi.nlm.nih.gov/28394818/

40. Rogasch JMM, Albers J, Steinkrüger FL, et al. Point Spread Function Reconstruction for Integrated 18F-FET PET/MRI in Patients With Glioma: Does It Affect SUVs and Respective Tumor-toBackground Ratios? Clin Nucl Med [Internet]. Lippincott Williams and Wilkins; 2019 [cited 2021];44:e280-e285. Available from: https://pubmed.ncbi.nlm.nih.gov/30562198/

41. Hudson HM, Larkin RS. Accelerated Image Reconstruction Using Ordered Subsets of Projection Data. IEEE Trans Med Imaging [Internet]. IEEE Trans Med Imaging; 1994 [cited 2020];13:601-609. Available from: https://pubmed.ncbi.nlm. nih.gov/18218538/

42. Jeih L, Strother SC. The convergence of object dependent resolution in maximum likelihood based tomographic image reconstruction. Phys Med Biol [Internet]. Phys Med Biol; 1993 [cited 2020];38:55-70. Available from: https://pubmed.ncbi.nlm.nih. gov/8426869/

43. Qi J, Leahy RM. Iterative reconstruction techniques in emission computed tomography [Internet]. Phys. Med. Biol. Phys Med Biol; 2006 [cited 2020]. Available from: https://pubmed.ncbi. nlm.nih.gov/16861768/

44. Vandenberghe S, Moskal P, Karp JS. State of the art in total body PET. EJNMMI Phys. Springer; 2020.

45. Sekine T, Delso G, Zeimpekis KG, et al. Reduction of 18F-FDG dose in clinical PET/MR imaging by using silicon photomultiplier detectors. Radiology. Radiological Society of North America Inc.; 2018;286:249-259.

46. Queiroz MA, Delso G, Wollenweber S, et al. Dose optimization in TOF-PET/MR compared to TOF-PET/CT. PLoS One. Public Library of Science; $2015 ; 10$.

47. Hatami S, Frye SA, McMunn A, et al. Added Value of Digital Over Analog PET/CT: More Significant as Image Field of View (FOV) and Body Mass Index (BMI) Increases. J Nucl Med Technol [Internet]. Society of Nuclear Medicine; 2020 [cited 2020];jnmt.120.244160. Available from: https://pubmed.ncbi. nlm.nih.gov/32887763/

48. Ahn S, Fessler JA. Globally convergent image reconstruction for emission tomography using relaxed ordered subsets algorithms. IEEE Trans Med Imaging. 2003;22:613-626.

49. Schwyzer M, Ferraro DA, Muehlematter UJ, et al. Automated detection of lung cancer at ultralow dose PET/CT by deep neural networks - Initial results. Lung Cancer. Elsevier Ireland Ltd; 2018;126:170-173.

50. Aljared A, Alharbi AA, Huellner MW. BSREM Reconstruction for Improved Detection of In-Transit Metastases with Digital FDG-PET/CT in Patients with Malignant Melanoma. Clin Nucl Med. Lippincott Williams and Wilkins; 2018;43:370-371.

51. Nguyen NC, Vercher-Conejero JL, Sattar A, et al. Image quality and diagnostic performance of a digital pet prototype in patients with oncologic diseases: Initial experience and comparison with analog PET. J Nucl Med. Society of Nuclear Medicine Inc.; 2015;56:1378-1385.

52. Gnesin S, Kieffer C, Zeimpekis K, et al. Phantom-based image quality assessment of clinical 18F-FDG protocols in digital PET/ CT and comparison to conventional PMT-based PET/CT. EJNMMI Phys [Internet]. Springer; 2020 [cited 2020];7. Available from: https://pubmed.ncbi.nlm.nih.gov/31907664/

53. Liberini V, Kotasidis F, Treyer V, et al. Impact of PET data driven respiratory motion correction and BSREM reconstruction of 68Ga-DOTATATE PET/CT for differentiating neuroendocrine tumors (NET) and intrapancreatic accessory spleens (IPAS). Sci Rep [Internet]. Nature Publishing Group; 2021 [cited 2021];11:2273. Available from: http://www.nature.com/articles/ s41598-020-80855-4

54. Baratto L, Duan H, Ferri V, et al. The Effect of Various $\beta$ Values on Image Quality and Semiquantitative Measurements in 68Ga-RM2 and 68Ga-PSMA-11 PET/MRI Images Reconstructed with a Block Sequential Regularized Expectation Maximization Algorithm. Clin Nucl Med [Internet]. Lippincott Williams and Wilkins; 2020 [cited 2021];45:506-513. Available from: https://pubmed.ncbi.nlm.nih. gov/32433170/

55. McHugh ML. Interrater reliability: The kappa statistic. Biochem Medica [Internet]. Biochemia Medica, Editorial Office; 2012 [cited 2020];22:276-282. Available from: /pmc/articles/ PMC3900052/?report=abstract

56. IBM Corp. Released 2017. IBM SPSS Statistics for Windows, Version 25.0. Armonk, NY: IBM Corp.

57. 58. Teoh EJ, McGowan DR, Bradley KM, et al. Novel penalised likelihood reconstruction of PET in the assessment of histologically verified small pulmonary nodules. Eur Radiol. Springer Verlag; 2016;26:576-584.

58. 59. Lindström E, Velikyan I, Regula N, et al. Regularized reconstruction of digital time-of-flight 68Ga-PSMA-11 PET/CT for the detection of recurrent disease in prostate cancer patients. Theranostics. 2019;9:3476-3484.

59. Lindström E, Lindsjö L, Sundin A, et al. Evaluation of blocksequential regularized expectation maximization reconstruction of $68 \mathrm{Ga}$-DOTATOC, $18 \mathrm{~F}$-fluoride, and 11C-acetate whole-body examinations acquired on a digital time-of-flight PET/CT scanner. EJNMMI Phys [Internet]. Springer; 2020 [cited 2020];7. Available from: https://pubmed.ncbi.nlm.nih.gov/32542512/

60. Shkumat NA, Vali R, Shammas A. Clinical evaluation of reconstruction and acquisition time for pediatric 18F-FDG brain PET using digital PET/CT. Pediatr Radiol [Internet]. Springer; 2020 [cited 2020];50:966-972. Available from: https://pubmed.ncbi. nlm.nih.gov/32125447/

61. Caribé PRRV, Koole M, D'Asseler Y, et al. Noise reduction using a Bayesian penalized-likelihood reconstruction algorithm on a time-of-flight PET-CT scanner. EJNMMI Phys [Internet]. Springer; 2019 [cited 2020];6:22. Available from: http://www. ncbi.nlm.nih.gov/pubmed/31823084

62. Diaz ME, Debowski M, Hukins C, et al. Non-small cell lung cancer brain metastasis screening in the era of positron emission tomography-CT staging: Current practice and outcomes. J Med Imaging Radiat Oncol [Internet]. Blackwell Publishing; 2018 [cited 2020];62:383-388. Available from: http://doi.wiley. com/https://doi.org/10.1111/1754-9485.12732

63. Silvestri GA, Gonzalez A V., Jantz MA, et al. Methods for staging non-small cell lung cancer: Diagnosis and management of lung cancer, 3rd ed: American college of chest physicians evidencebased clinical practice guidelines. Chest [Internet]. Chest; 2013 [cited 2020];143. Available from: https://pubmed.ncbi.nlm.nih. gov/23649440/

64. Milano MT, Bates JE, Budnik J, et al. Risk of brain metastases in T1-3NO NSCLC: a population-based analysis. Lung Cancer Manag [Internet]. Future Medicine Ltd; 2020 [cited 2020];9:LMT25. Available from: /pmc/articles/PMC7110582/?report=abstract

65. Waqar SN, Samson PP, Robinson CG, et al. Non-small-cell Lung Cancer With Brain Metastasis at Presentation. Clin Lung Cancer [Internet]. Elsevier Inc.; 2018 [cited 2020];19:e373-e379. Available from: https://pubmed.ncbi.nlm.nih.gov/29526531/

Publisher's Note Springer Nature remains neutral with regard to jurisdictional claims in published maps and institutional affiliations. 